\title{
Synthesis and Application of a 5'-Aldehyde Phosphoramidite for Covalent Attachment of DNA to Biomolecules
}

\author{
Chandrasekhar V. Miduturu and Scott K. Silverman* \\ Department of Chemistry, University of Illinois at Urbana-Champaign, 600 South Mathews Avenue, Urbana, Illinois 61801
}

\section{Table of Contents}

General experimental procedures. page S1

Tabulation of ${ }^{1} \mathrm{H}$ and ${ }^{13} \mathrm{C}$ NMR spectral peaks for compounds 3-7. page S2

Images of ${ }^{1} \mathrm{H}$ NMR spectra for compounds 3-7 pages S3-S7 Image of ${ }^{31} \mathrm{P}$ NMR spectrum for compound 7 page S8

General Experimental Procedures. Reagents were commercial grade and used without purification unless otherwise indicated. Dry solvents were obtained from sealed bottles (pyridine, $\mathrm{MeOH}$ ) or by passage through activated neutral alumina under positive argon pressure $\left(\mathrm{CH}_{2} \mathrm{Cl}_{2}\right.$, THF). All reactions were performed at room temperature under argon or nitrogen unless otherwise noted. A reaction temperature of $0{ }^{\circ} \mathrm{C}$ was maintained with an ice-water bath. Thin-layer chromatography (TLC) was performed on silica gel plates pre-coated with fluorescence indicator with visualization by UV light (254 nm). Organic solutions that were dried over $\mathrm{Na}_{2} \mathrm{SO}_{4}$ were filtered through paper to remove the drying agent, and the paper was rinsed into the collection flask with an appropriate organic solvent. All chromatographic purifications used 230-400 mesh silica gel. NMR spectra were obtained at the indicated frequencies. ${ }^{1} \mathrm{H}$ and ${ }^{13} \mathrm{C}$ chemical shifts in parts per million $(\delta)$ were referenced to internal tetramethylsilane (TMS). The designation “ABq" for a ${ }^{1} \mathrm{H}$ NMR peak indicates that the particular peak was one partner of an $\mathrm{AB}$ quartet; if additional splittings were evident, they are noted following the $\mathrm{ABq}$ designation (e.g., ABqd). In the ${ }^{1} \mathrm{H}$ NMR spectra, peaks not assigned to the indicated compound are labeled as follows: $\mathrm{w}=$ water; $\mathrm{n}=\mathrm{NMR}$ solvent; $\mathrm{s}=$ common solvent (e.g., $\mathrm{CH}_{2} \mathrm{Cl}_{2}$ or EtOAc). ${ }^{31} \mathrm{P}$ chemical shifts in $\delta$ were referenced to external $\mathrm{H}_{3} \mathrm{PO}_{4}$ at $0 \mathrm{ppm}$. 


\section{Tabulation of ${ }^{1} \mathrm{H}$ and ${ }^{13} \mathrm{C}$ NMR spectral peaks for compounds 3-7}

5'-O-Allyl-3'-O-benzylthymidine (3): ${ }^{1} \mathrm{H}$ NMR (400 MHz, $\mathrm{CDCl}_{3}$ ) $\delta 8.89$ (br s, $\left.1 \mathrm{H}\right), 7.63$ (br q, $1 \mathrm{H}, J$ $=1.0 \mathrm{~Hz}$ ), 7.39-7.28 (m, 5H), 6.38 (dd, $1 \mathrm{H}, J=6.0 \mathrm{~Hz}), 5.89$ (ddt, $1 \mathrm{H}, J=15.0,10.5,5.0 \mathrm{~Hz}), 5.27$ (dq, $1 \mathrm{H}, J=17.0,1.5 \mathrm{~Hz}$ ), 5.22 (dq, $1 \mathrm{H}, J=10.2,1.5 \mathrm{~Hz}), 4.51$ and 4.57 (ABq, $1 \mathrm{H}, J=11.6 \mathrm{~Hz}), 4.25$ (dt, $1 \mathrm{H}, J=6.0,2.5 \mathrm{~Hz}$ ), 4.23 (q, 1H, $J=2.5 \mathrm{~Hz}$ ), $4.04(\mathrm{~m}, 2 \mathrm{H}), 3.71$ and 3.62 (ABqd, $2 \mathrm{H}, J=10.6,2.0$ Hz), 2.47 (ddd, 1H, $J=13.2,5.6,2.4 \mathrm{~Hz}$ ), 2.10 (ddd, 1H, $J=13.2,7.6,5.2 \mathrm{~Hz}$ ), 1.89 (d, 3H, $J=1.0$ $\mathrm{Hz}) ;{ }^{13} \mathrm{C}$ NMR $\left(125 \mathrm{MHz}, \mathrm{CDCl}_{3}\right) \delta 163.8,150.3,137.5,135.8,133.9,128.5,127.6,117.5,110.8,85.1$, 83.9, 79.2, 72.3, 71.3, 70.4, 37.9, 12.5 .

5'-O-(2,3-Dihydroxypropyl)-3'-O-benzylthymidine (4): ${ }^{1} \mathrm{H}$ NMR (400 $\left.\mathrm{MHz}, \mathrm{CDCl}_{3}\right) \delta 8.62$ (br s, $1 \mathrm{H}), 7.41-7.29(\mathrm{~m}, 6 \mathrm{H}), 6.25(\mathrm{~m}, 1 \mathrm{H}), 4.54(\mathrm{ABq}, 2 \mathrm{H}, J=11.6 \mathrm{~Hz}), 4.21(\mathrm{dt}, 1 \mathrm{H}, J=6.0,2.5 \mathrm{~Hz}), 4.19$ (q, 1H, $J=3.0 \mathrm{~Hz}$ ), 3.89 (m, 1H), 3.78-3.52 (m, 6H), 2.73 (br s, 1H), 2.46 (m, 1H), 2.28 (br s, $1 \mathrm{H}), 2.14$ $(\mathrm{m}, 1 \mathrm{H}), 1.92$ (d, 3H, $J=1.0 \mathrm{~Hz}) ;{ }^{13} \mathrm{C} \mathrm{NMR}\left(125 \mathrm{MHz}, \mathrm{CDCl}_{3}\right) \delta 164.4,151.0,137.0,136.0,128.6$, 128.0, 127.7, 110.0, 85.8, 83.6, 78.6, 72.9, 71.5, 71.3, 70.7, 64.0, 38.2, 12.7.

5'-O-(2,3-Di-O-benzoylpropyl)- $\boldsymbol{N}^{\mathbf{3}}$-benzoyl-3'-O-benzylthymidine (5): ${ }^{1} \mathrm{H}$ NMR (400 MHz, $\left.\mathrm{CDCl}_{3}\right) \delta$ 8.60-8.05 and 7.95-7.91 (m, 6H) (mixture), 7.65-7.42 (m, 7H) (mixture), 7.34-7.20 (m, 5H) (mixture), 6.31 (dd, $1 \mathrm{H}, J=6.0 \mathrm{~Hz}$ ) (minor), 6.29 (dd, $1 \mathrm{H}, J=6.0 \mathrm{~Hz}$ ) (major), $5.73(\mathrm{~m}, 1 \mathrm{H})$ (minor), $5.66(\mathrm{~m}, 1 \mathrm{H})$ (major), 4.69-4.55 (m, 2H) (mixture), 4.46 (ABq, 2H, $J=11.6 \mathrm{~Hz}$ ) (major), 4.35 (ABq, 2H, $J=11.6$ $\mathrm{Hz}$ ) (minor), 4.21 (m, 2H) (major), 4.17 (m, 1H, $J=3.0 \mathrm{~Hz}$ ) (minor), 4.09 (m, 1H) (minor), 3.98-3.86 (m, 1H), 3.86-3.52 (m, 3H), 2.46 (ddd, $1 \mathrm{H}, J=13.2,5.6,2.4 \mathrm{~Hz}$ ) (major), 2.39 (ddd, $1 \mathrm{H}, J=13.2,5.6$, $2.4 \mathrm{~Hz}$ ) (minor), $2.06(\mathrm{~m}, 1 \mathrm{H})$ (mixture of diastereomers), 1.93 (d, 3H, $J=1.0 \mathrm{~Hz}$ ) (minor diastereomer), 1.92 (d, 3H, $J=1.0 \mathrm{~Hz}$ ) (major diastereomer); ${ }^{13} \mathrm{C}$ NMR $\left(125 \mathrm{MHz}, \mathrm{CDCl}_{3}\right) \delta 169.0$, 166.1 , 162.8, 150.0, 135.2, 135.0, 133.6, 133.4, 130.5, 129.70, 129.67, 129.1, 127.9, 127.7, 128.6, $128.5,110.5,85.4,83.8,78.9,71.6,71.5,70.5,69.9,63.2,37.8,12.7$.

5'-O-(2,3-Di-O-benzoylpropyl)- $\boldsymbol{N}^{\mathbf{3}}$-benzoylthymidine (6): ${ }^{1} \mathrm{H}$ NMR (500 $\left.\mathrm{MHz}, \mathrm{CDCl}_{3}\right) \delta$ 8.06-8.00 and 7.94-7.88 (m, 6H) (mixture), 7.65-7.54 and 7.50-7.41 (m, 7H) (mixture), $6.30(\mathrm{t}, 1 \mathrm{H}, J=6.0 \mathrm{~Hz})$ (minor), 6.25 (t, 1H, $J=6.0 \mathrm{~Hz}$ ) (major), 5.71 (m, 1H) (mixture), 4.66 (d, 2H, $J=4.8 \mathrm{~Hz}$ ) (mixture), $4.43(\mathrm{~m}, 1 \mathrm{H})$ (mixture), 4.08-3.68 (m, 5H) (mixture), 2.71 (br s, 1H) (major), 2.42 (br s, 1H) (minor), 2.35-2.22 (m, 1H) (mixture), 2.20-2.12 (m, 1H) (mixture of diastereomers), 1.92 (d, 3H, $J=1.2 \mathrm{~Hz}$ ) (minor diastereomer), $1.90\left(\mathrm{~d}, 3 \mathrm{H}, J=1.2 \mathrm{~Hz}\right.$ ) (major diastereomer); ${ }^{13} \mathrm{C}$ NMR (125 MHz, $\left.\mathrm{CDCl}_{3}\right) \delta$ 169.0, 166.2, 162.8, 149.2, 135.4, 135.0, 133.7, 133.5, 131.5, 130.4, 129.7, 129.3, 129.1, 128.6, 128.5, 111.0, 85.1, 84.9, 70.7, 70.4, 70.3, 69.8, 63.0, 40.4, 12.7.

\section{5'-O-(2,3-Di-O-benzoylpropyl)- $\boldsymbol{N}^{3}$-benzoylthymidine-3'-O-(2-cyanoethyl- $\boldsymbol{N}, \boldsymbol{N}$-diisopropyl)-}

phosphoramidite (7): ${ }^{1} \mathrm{H}$ NMR (400 MHz, acetone- $\left.d_{6}\right) \delta 8.10-7.98(\mathrm{~m}, 6 \mathrm{H})$ (mixture), 7.83, 7.81, 7.79 and 7.78 (br d, $1 \mathrm{H}, J=1.0 \mathrm{~Hz}$ ) (4 diastereomers), 7.76-7.72 and 7.68-7.48 (m, 8H) (mixture), 6.31 (m, 1H) (mixture), 5.86-5.71 (m, 1H) (mixture), 4.82-4.58 (m, 3H) (mixture), 4.32, 4.29, 4.23, 4.22 (q, 1H, $J=3.0 \mathrm{~Hz}$ ) (4 diastereomers), 4.14-3.72 (m, 6H) (mixture), 3.63 (m, 2H) (mixture), $2.76(\mathrm{~m}, 2 \mathrm{H})$ (mixture), 2.52-2.30 (m, 2H) (mixture), 1.88 (m, 3H) (mixture), 1.17 (m, 12H) (mixture of diastereomers); ${ }^{13} \mathrm{C}$ NMR (125 MHz, acetone- $\left.d_{6}\right) \delta$ 169.7, 165.9, 162.7, 149.7, 136.4, 135.2, 133.6, 133.5, 132.3, 130.5, 129.7, 129.6, 129.4, 128.8, 119.0, 110.5, 85.7, 85.4, 74.3, 71.2, 70.4, 67.9, 63.6, 58.6, 43.2, 39.5, 24.1, 20.0, 12.1. 


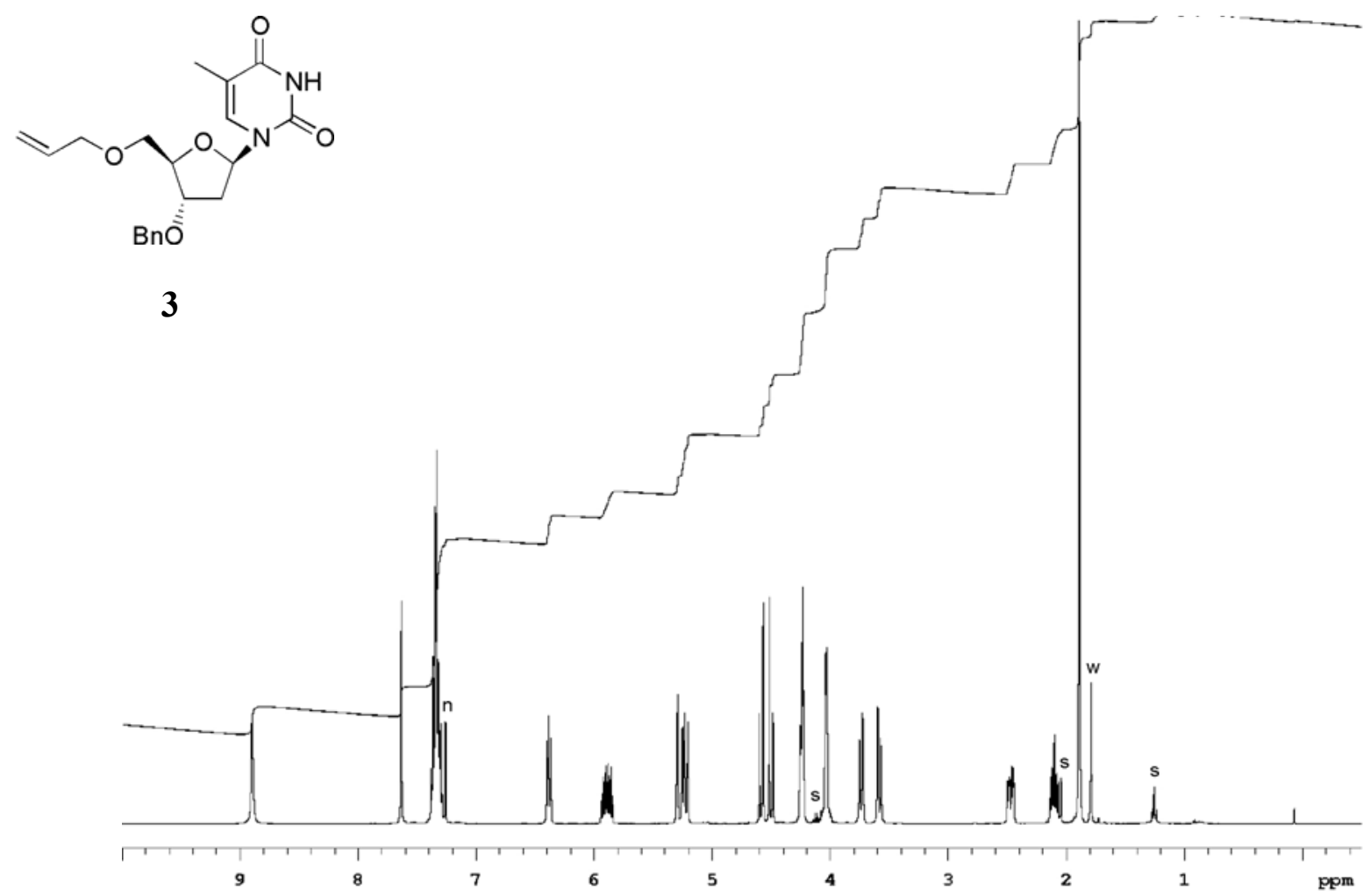




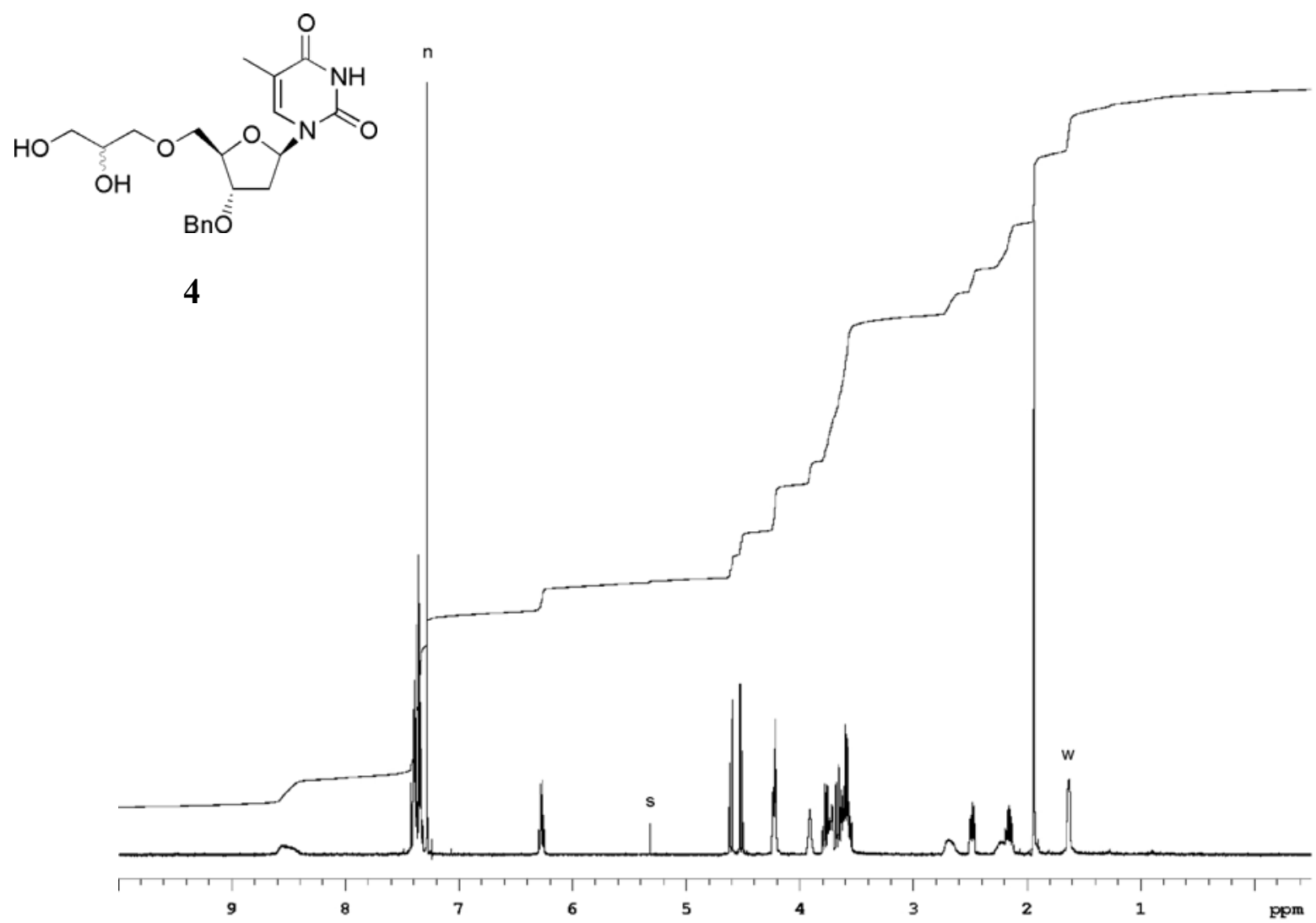




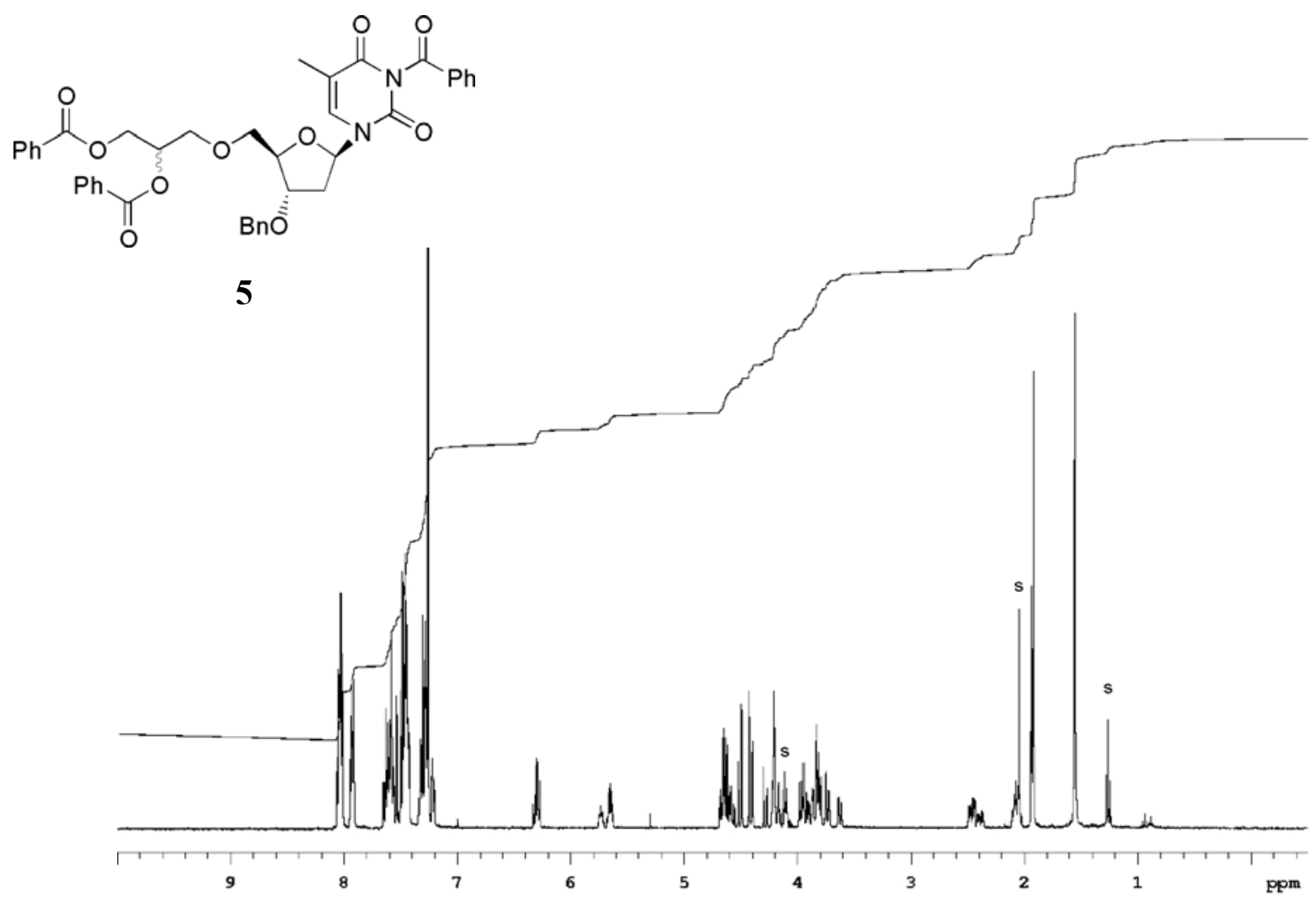




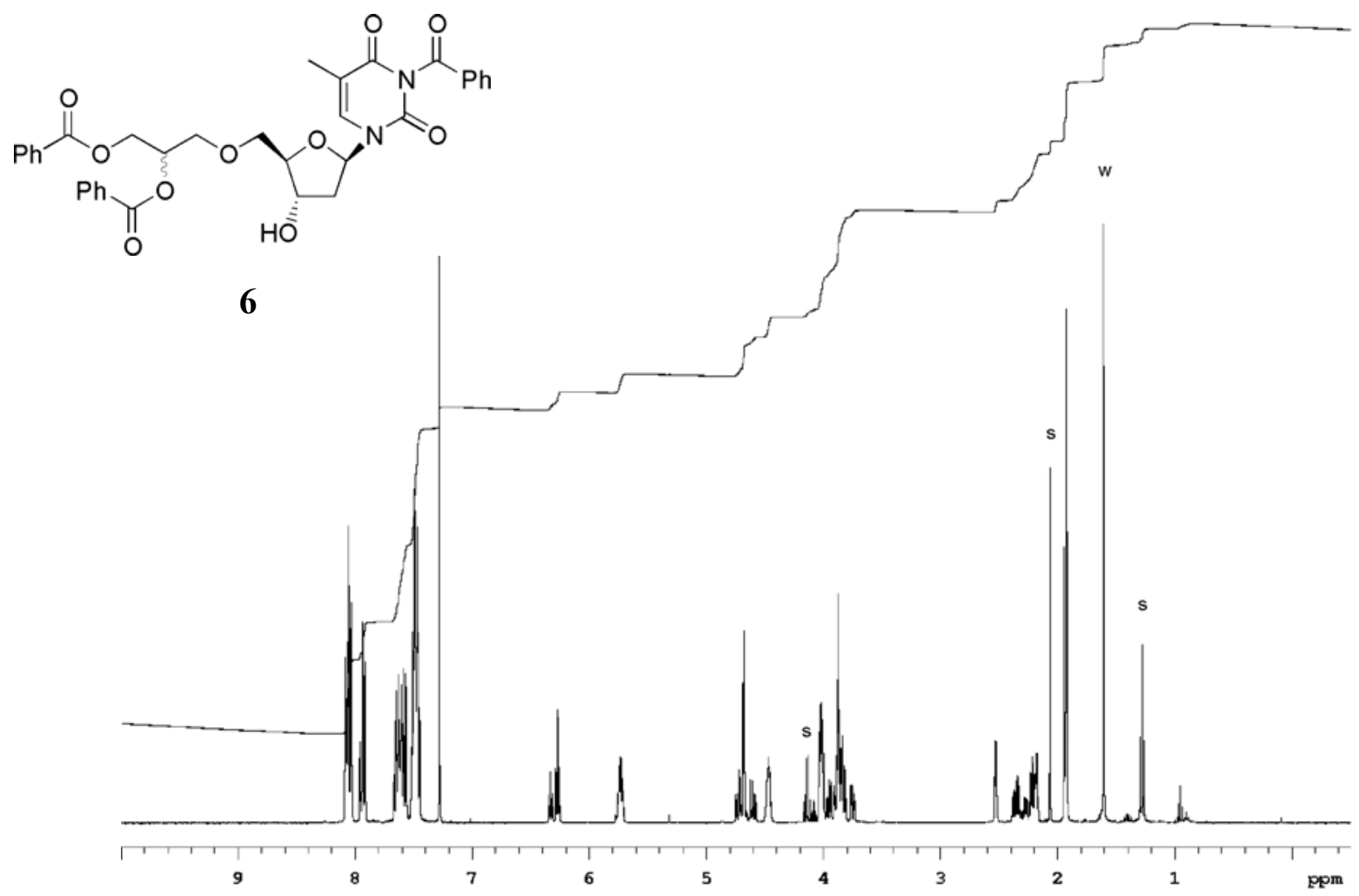




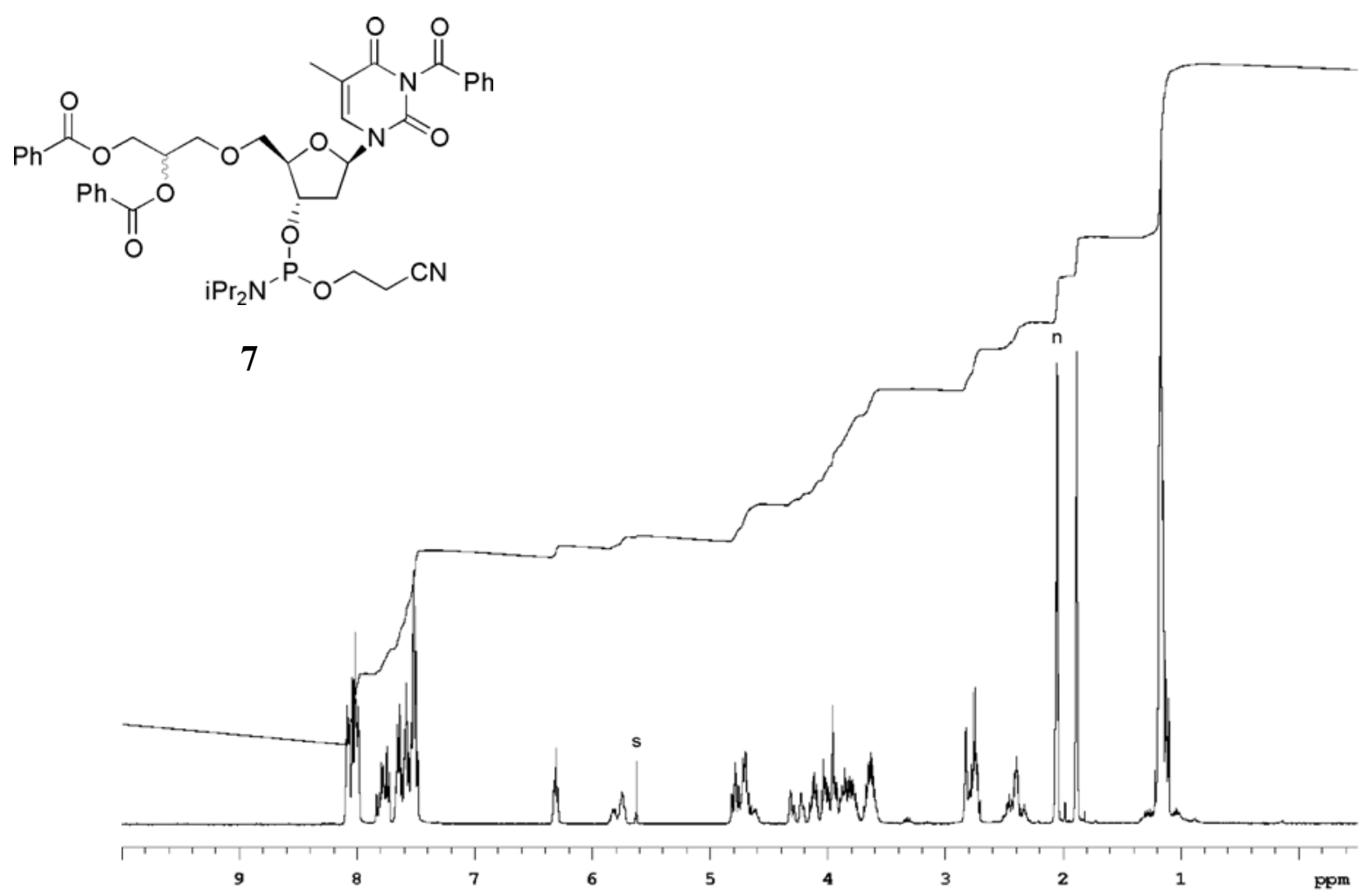


<smiles>Cc1cn(C2CC(OP(NC(C)C)OCCC#N)C(COCC(COC(=O)c3ccccc3)OC(=O)c3ccccc3)O2)c(=O)n(C(=O)c2ccccc2)c1=O</smiles>

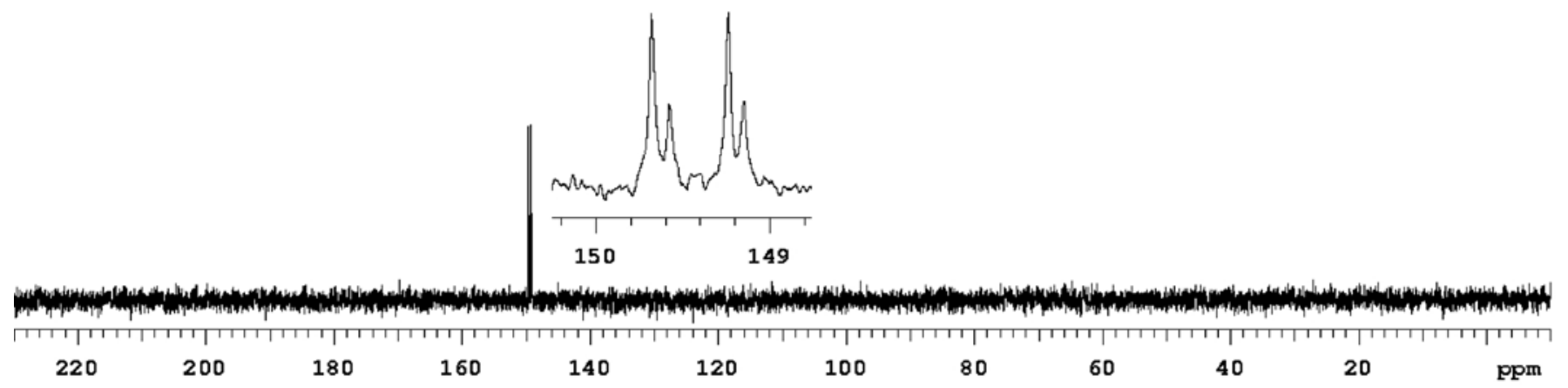

